\title{
O enfrentamento do câncer fora de possibilidade terapêutica: uma revisão integrativa
}

\section{Coping with cancer out of therapeutic possibility and its impact on nursing care interventions: an integrative review}

\begin{abstract}
Wanderson Paiva dos Santos' • Francisca de Kassia Ferreira da Silva ${ }^{2}$ Isadora da Silva Ribeiro ${ }^{3} \bullet$ Aline Isabella Saraiva Costa de Souza Abreu ${ }^{4} \bullet$ Mariana Rodrigues da Silva de Menezes ${ }^{5} \bullet$ Maria Luiza Rêgo Bezerra $^{6}$
\end{abstract}

\begin{abstract}
RESUMO
Objetivou-se identificar quais são as principais estratégias de enfrentamento apresentadas por pacientes em cuidados paliativos oncológicos. Trata-se de uma Revisão integrativa nas bases de dados MEDLINE, BDENF - Enfermagem, LILACS e IBECS - ES em artigos nos idiomas inglês, espanhol e português, no período de 2015 a 20I9, selecionando-se II artigos por meio de leitura exploratória e crítica dos títulos, resumos e resultados alcançados nos estudos. Foram identificadas estratégias de enfrentamento, tais como enfrentamento familiar, religioso, autoculpa e adaptação, com prioridade ao enfrentamento religioso. No entanto, houve predominância do coping religioso positivo. Os estudos apresentaram diferenças em relação à metodologia e instrumentos utilizados. Emergiram categorias de enfrentamento positivo, negativo e recursos relacionados à rede social de apoio do paciente. A religião/espiritualidade e a rede social de apoio foram as principais estratégias de enfrentamento apresentadas pelos pacientes em cuidados paliativos oncológicos.
\end{abstract}

Descritores: Cuidados Paliativos. Neoplasias. Adaptação Psicológica.

\begin{abstract}
The objective was to identify which are the main coping strategies presented by patients in palliative oncology care. It is an integrative review in the databases MEDLINE, BDENF - Enfermagem, LILACS and IBECS - ES in articles in English, Spanish and Portuguese, in the period from 2015 to 2019, selecting II articles through exploratory reading and criticism of the titles, abstracts and results achieved in the studies. Coping strategies were identified, such as family, religious, self-guilt and adaptation, with priority to religious coping. However, there was a predominance of positive religious coping. The studies showed differences in relation to the methodology and instruments used. Categories of positive, negative coping and resources related to the patient's social support network emerged. Religion / spirituality and the social support network were the main coping strategies presented by patients in palliative oncology care.
\end{abstract}

Keywords: Palliative Care. Neoplasms. Psychological Adaptation.

I Enfermeiro. Especialista em Cuidados Paliativos. Docente no Instituto Técnico Madre Teresa.

2 Acadêmica enfermagem no Centro Universitário do Distrito Federal - UDF.

3 Enfermeira. Pós-graduanda em Ginecologia.

4 Doutora em Psicologia clínica. Enfermeira. Docente no Centro Universitário do Distrito Federal - UDF.

5 Mestre em saúde ambiente e sociedade. Enfermeira. Docente do Centro Universitário do Distrito Federal.

6 Doutoranda em Bioética (UnB). Mestre em Enfermagem (UnB). Especialista em Gestão em Saúde Pública (INESPO). Enfermeria (UFMA). Docente do Curso de Enfermagem da Universidade Paulista (UNIP), campus Brasília. 


\section{INTRODUÇÃO}

O câncer é uma doença crônico-degenerativa, caracterizada pelo crescimento desordenado das células, multiplicando-se rapidamente e atingindo tecidos e órgãos adjacentes sendo considerado um grave problema de saúde pública. $O$ câncer possui grande amplitude epidemiológica e é considerado a segunda causa de morte no Brasil (1).

A doença oncológica produz vários sinais e sintomas indesejados, tais como: fadiga, sangramento, náuseas, vômitos e dor, sendo este último frequentemente relatado pelos pacientes para expressar o sofrimento causado pela patologia. $O$ diagnóstico do câncer traz consigo, não apenas sintomas físicos já citados, mas a constatação da instalação de uma doença de alta morbidade, a vivência da dor, a alteração da autoimagem, a possibilidade de morte e o distanciamento da família e das atividades laborais, além do sofrimento emocional, que aumenta à medida que a doença avança ${ }^{(2)}$.

As neoplasias podem atingir um nível de complexidade onde não há mais possibilidade de cura, sendo necessária a utilização dos denominados cuidados paliativos. Esse tipo de abordagem busca garantir qualidade de vida, proporcionando conforto ao paciente oncológico e seus familiares e controlando os sinais e sintomas da doença ${ }^{(3-4)}$.

Neste âmbito, é comum que os pacientes procurem adotar diferentes comportamentos com o objetivo de lutar contra a doença oncológica. Diversas estratégias de enfrentamento são analisadas para que se ajude a pessoa com câncer a suportar a enfermidade, podendo sofrer alterações/adaptações de indivíduo para indivíduo ${ }^{(5)}$.

O enfrentamento ou coping é um grupo de estratégias para lidar com algo que é percebido pelo indivíduo como uma ameaça iminente, uma sobrecarga às suas capacidades cognitivas e comportamentais do momento. É compreendido a partir das estratégias com foco no problema e das estratégias centradas na emoção. As focadas no problema objetivam analisar e conceituar a situação vivenciada e encontrar alternativas para resolvê-la, enquanto as estratégias centradas na emoção referem-se às atitudes adotadas pelos indivíduos quando notam que os estressores não podem ser modificados, sendo necessário continuar interagindo com eles ${ }^{(6)}$.

As estratégias de enfrentamento são evidenciadas por sentimentos de esperança, positividade ou negação da situação e das consequências ocasionadas. Podem ser positivas ou negativas, a depender da experiência de cada paciente, onde o indivíduo tenta extrair subsídios dos acontecimentos, no intuito de diminuir o transtorno emocional provocado por uma situação estressante ${ }^{(6)}$.

Nesse sentido, é importante compreender as pessoas em sua multidimensionalidade nos aspectos biológicos, psicológicos, sociais e espirituais propiciando a integralidade, com respeito a sua subjetividade, princípios e cren- ças pessoais e familiares. Tais aspectos exercem grande influência durante a paliação, no sentido de fornecer suporte estrutural ${ }^{(7)}$.

O cuidado da equipe de enfermagem, sobretudo, por meio da implementação da Sistematização da Assistência de Enfermagem (SAE) frente ao paciente oncológico, é complexo e deve ser altamente especializado, visando prevenir complicações, reduzindo incapacidades e procurando a sua recuperação e qualidade de vida. Dessa forma, a equipe de enfermagem busca garantir intervenções que englobam a multidimensionalidade do indivíduo, realizando ações compatíveis com a realidade de cada paciente, em busca de um resultado satisfatório ou que melhore a qualidade de vida relacionada ao câncer ${ }^{(8-10)}$.

Nesse raciocínio, entende-se que os enfermeiros devem conhecer/reconhecer as inter-relações entre as dimensões humanas e, assim, proporcionar um cuidado integral e efetivo ao paciente oncológico, propiciando uma assistência contínua, seja curativa ou paliativa (II).

No entanto, para que o enfermeiro possa intervir com eficácia em um quadro oncológico paliativo, é preciso atentar-se aos sinais apresentados pelo paciente. Sendo necessário levar em consideração aspectos não apenas biológicos, mas também psicossociais, que poderão cooperar com o manejo adequado oferecido pelo enfermeiro através das Intervenções de Enfermagem ${ }^{(12)}$.

Desta forma, a partir da significância ora descrita objetivou-se identificar as principais estratégias de enfrentamento apresentadas pelos pacientes oncológicos em cuidados paliativos.

\section{METODOLOGIA}

Trata-se de uma revisão integrativa, valendo-se da estratégia PICO, acrônimo para Paciente, Intervenção, Comparação e Outcomes (desfechos), que se baseia na elaboração de uma pergunta norteadora para guiar a busca de evidências ${ }^{(13)}$.

$\mathrm{Na}$ construção do presente estudo, foram realizadas as seguintes etapas: I) Seleção da pergunta norteadora; 2) Determinação dos critérios de inclusão e exclusão e busca na literatura; 3) Determinação das informações a serem extraídas e categorização dos estudos; 4)Avaliação dos estudos incluídos na revisão integrativa; 5) Interpretação dos resultados e; 6) A apresentação da revisão com síntese do conhecimento produzido ${ }^{(14)}$.

Desse modo, a estratégia PICO foi empregada conferindo-se: a $\mathrm{P}$, os pacientes em cuidados paliativos; ao I, as principais formas de enfrentamento do câncer; ao C, a comparação com os resultados obtidos nos estudos; e ao $\mathrm{O}$, o resultado esperado na pesquisa ${ }^{(13)}$. Nesse sentido, a pergunta definida foi: "Quais são as principais formas de enfrentamento do câncer apresentadas por pacientes oncológicos em cuidados paliativos?". 
Para isso, realizou-se uma busca nas seguintes bases de dados: MEDLINE, BDENF - Enfermagem, LILACS e IBECS - ES, utilizando os descritores indexados "Cuidados Paliativos, Neoplasias e Adaptação Psicológica" e seus correspondentes em inglês "Palliative Care, Neoplasms e Psychological Adaptation" por meio do cruzamento: Adaptação Psicológica AND Cuidados Paliativos AND Neoplasias.

Ressalta-se a delimitação nos últimos cinco anos, a partir de 2015, para a estratégia de busca. Os critérios de inclusão foram: estudos disponíveis na íntegra, em que a população era constituída por pacientes adultos em cuidados paliativos; estudos publicados nas línguas portuguesa, inglesa ou espanhola, registrados nas bases de dados até outubro de 2019.

Foram excluídos os estudos com menores de 18 anos ou que não contemplavam a tríade temática "enfrentamento do câncer, cuidados paliativos e intervenções de enfermagem", assim como os que abordavam o "luto" e "oncologia pediátrica" (dada especificidade da população), dissertações de mestrado, teses de doutorado, editoriais, anais de congresso, guidelines e estudos que abordavam a possibilidade terapêutica.

Inicialmente foram identificados 486 artigos condizentes com o tema. Após realizar o recorte temporal e selecionar os estudos que estavam disponíveis na íntegra, o montante resultou em 108 artigos, sendo que 9 estavam repetidos nas bases de dados verificadas e 85 foram excluídos por estarem alinhados com os critérios de exclusão. Dos 14 artigos selecionados, não foi possível ter acesso a 3, através Plataforma Capes Institucional, conforme fluxograma $\mathrm{I}$.
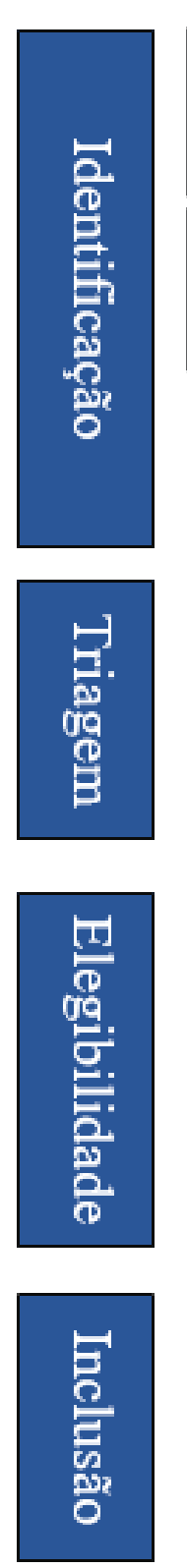
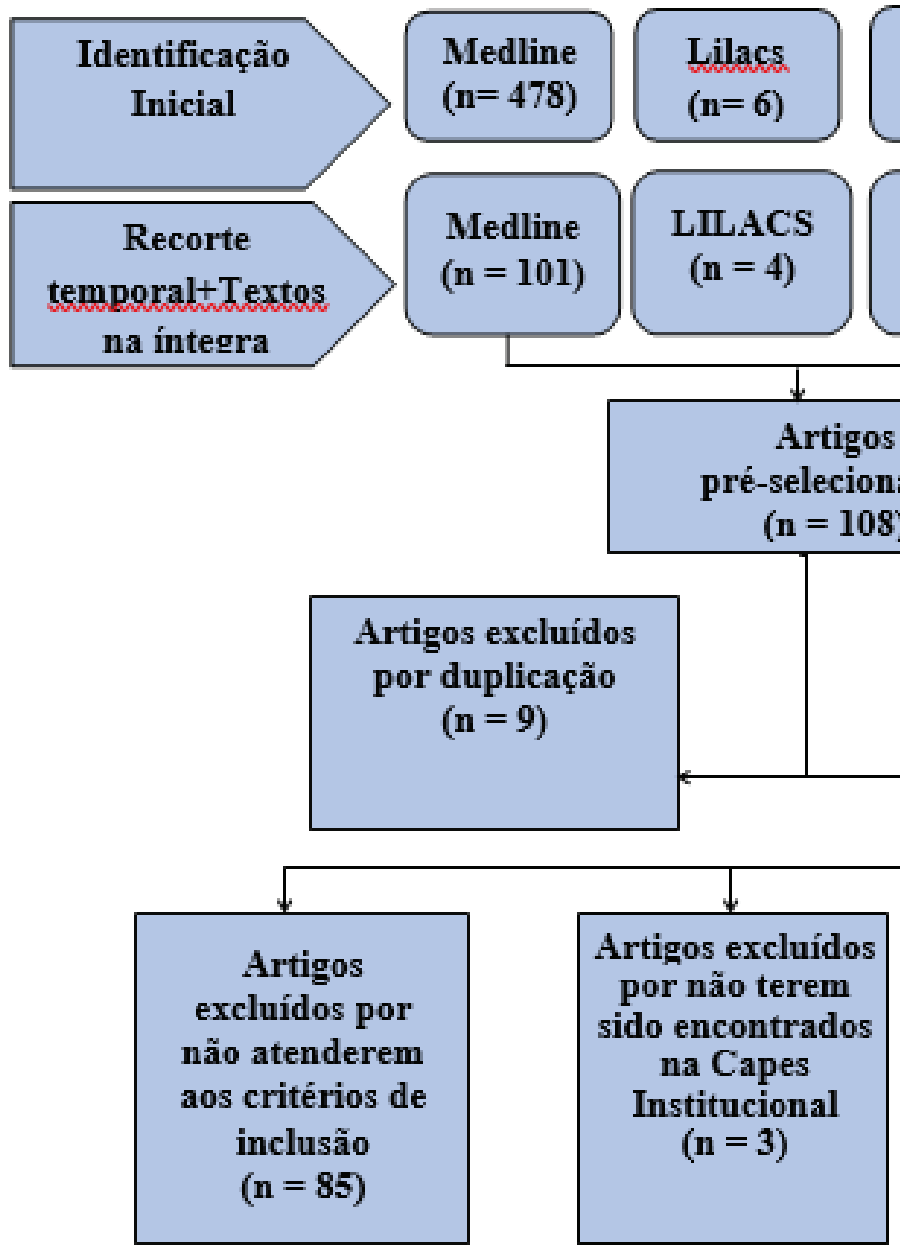

Artigos

excluídos por

não atenderem

aos critérios de

inclusão

$(\mathrm{n}=\mathbf{8 5})$

\section{Medline} $(n=478)$

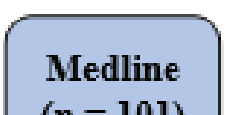

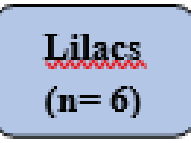

LILACS

$$
(n=4)
$$
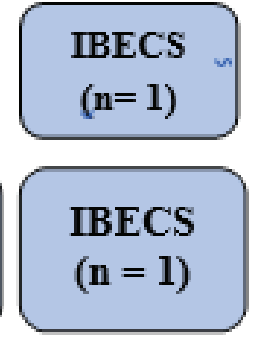

\section{BDENF}

$(n=2)$

\section{BDENF}

$(\mathrm{n}=2)$ 
Os artigos obtidos utilizaram metodologias diversas e dados primários, com os seguintes tipos de pesquisa: qualitativa (09), randomizado (02). Os artigos foram selecionados após a leitura do título e resumo, excluídos os duplicados, os que não contemplavam os critérios de inclusão e os que estavam de acordo com os de exclusão.

Foi realizada a leitura na íntegra dos artigos selecionados e os dados foram sistematizados por meio de fichamento contendo: nome dos autores, país de origem, objetivos, metodologia, população e principais resultados.

O processo de análise dos artigos ocorreu por meio da leitura exploratória e descritiva dos títulos, resumos e resultados encontrados sobre o enfrentamento do câncer em cuidados paliativos. A interpretação e a discussão foram realizadas de forma descritiva, em função do objetivo da pesquisa, o qual compete leitura na perspectiva qualitativa dos achados.

Após a leitura, foram verificados os níveis de evidência, os quais foram ordenados de acordo com a classificação dos estudos, sendo eles: Nível I - evidências provenientes de meta-análise de ensaios clínicos randomizados (ECR); Nível II - evidências resultantes de ensaios clínicos bem delineados sem randomização; Nível III - evidências oriundas de estudos descritivos e qualitativos; e Nível IV - evidências originárias de um único estudo descritivo ou qualitativo(15).

Por conseguinte, procedeu-se a apresentação da revisão com a síntese do conhecimento obtido descrevendo com clareza os principais aspectos resultantes do estudo. Os títulos e os resumos dos artigos identificados na busca eletrônica foram avaliados, de forma independente, por três revisores. Foi executada uma seleção dos estudos, conforme a questão norteadora e os critérios de inclusão e exclusão definidos previamente..

\section{RESULTADOS}

Após análise dos artigos encontrados na base de dados, foram contemplados para leitura II artigos, realizados em seis países, sendo que os Estados Unidos apresentaram maior número de publicações. Dez foram publicados na língua inglesa e um na língua portuguesa.

Os estudos selecionados foram realizados com pacientes oncológicos em cuidados paliativos. $\mathrm{O}$ número total de participantes dos estudos foi de 1399 pacientes, sendo que em um estudo houve participação de 96 voluntários saudáveis. Diversos instrumentos foram utilizados para avaliar as estratégias de enfrentamento. A variedade de instrumentos pode ser devida à diversidade de países envolvidos neste estudo. A respeito das características demográficas, a porcentagem de pacientes em cuidados paliativos do sexo feminino foi acima de $50 \%$. Quanto aos voluntários saudáveis, 64,6\% eram mulheres e $35,4 \%$, homens. Os achados dos estudos mostram diversas estratégias de enfrentamento apresentadas pelos pacientes em cuidados paliativos. No entanto, houve prevalência do enfrentamento espiritual com adoção de medidas que objetivam estar conectados a uma ligação de intimidade com um ser supremo.

As estratégias de enfrentamento foram classificadas em três subcategorias: estratégias de enfrentamento negativas, estratégias de enfrentamento positivas e recursos relacionados à rede social de apoio dos pacientes.

O foco das estratégias de enfrentamento negativas apresentado pelos pacientes em cuidados paliativos foi: coping religioso ou espiritual negativo, auto culpa e negação da condição atual e desengajamento comportamental ${ }^{(16-17)}$.

Com relação às estratégias de enfrentamento positivas foram identificados os seguintes focos: coping religioso ou espiritual, coping espiritual, coping religioso, aceitação, positividade não pensar na enfermidade e mudança de expectativas, ter espírito de luta, esquecimento cognitivo e fatalismo, aceitação e reenquadramento positivo, resiliência, reestruturação cognitiva e resistência para sobreviver e para ter uma boa morte ${ }^{(16 ; 17 ; 18 ; 19 ; 20 ; 21 ; 22 ; 23 ; 24 ; 25)}$

A respeito dos recursos relacionados à rede social, houve prioridade para aspectos como família, amigos e outros ambientes sociais (comunidade, bairro, vila), conexão com filhos e netos, não abandonando familiares, apoio do parceiro/cônjuge, sentindo-se parte de uma comunidade maior ${ }^{(22-23: 24)}$ apoio dos profissionais de saúde participantes ${ }^{(22 ; 23)}$, apoio emocional de outros ${ }^{(16 ; 17)}$.

Os artigos foram caracterizados quanto ao ano de publicação, país, autores, objetivos do estudo, metodologia, quantidade de participantes, principais resultados e nível de evidência, conforme quadro I. 
QUADRO1 - Caracterização dos artigos selecionados para a revisão integrativa. Brasília, DF, Brasil, 2019.

\begin{tabular}{|c|c|c|c|c|c|}
\hline $\begin{array}{c}\text { Autor/ano. } \\
\text { País }\end{array}$ & Objetivo(s) & Metodologia & População & Resumo dos resultados & NE \\
\hline $\begin{array}{l}\text { BOVERO, A. et al. } \\
\text { (2015), Estados } \\
\text { Unidos }\end{array}$ & $\begin{array}{l}\text { Comparar os } \\
\text { diferentes aspectos } \\
\text { da espiritualidade } \\
\text { nas últimas semanas } \\
\text { de vida de pacientes } \\
\text { com câncer avançado } \\
\text { internados em } \\
\text { manicômios e investigar } \\
\text { a QV, dor, ansiedade, } \\
\text { depressão e adaptação } \\
\text { psicológica frente ao } \\
\text { câncer. }\end{array}$ & $\begin{array}{l}\text { Estudo qualitativo, } \\
\text { utilizando entrevista } \\
\text { com escalas de } \\
\text { análise: a FACIT- } \\
\text { Sp-12, HADS, } \\
\text { EVA, Brief COPE e } \\
\text { FACT-G. }\end{array}$ & $\begin{array}{l}115 \text { pacientes } \\
\text { em suas últimas } \\
\text { semanas de } \\
\text { vida com câncer } \\
\text { avançado } \\
\text { internados em } \\
\text { manicômios. }\end{array}$ & $\begin{array}{l}\text { Trabalhadores e pacientes solteiros } \\
\text { com ensino superior expuseram QV } \\
\text { inferior, sendo que a espiritualidade } \\
\text { e busca da fé como apoio e suporte } \\
\text { foram associados positivamente } \\
\text { à QV e preparo para a morte, } \\
\text { enquanto ansiedade e dor foram } \\
\text { negativamente relacionados. }\end{array}$ & IV \\
\hline $\begin{array}{l}\text { MOSHER, C. E. et } \\
\text { al. (2015), Estados } \\
\text { Unidos. }\end{array}$ & $\begin{array}{l}\text { Detectar estratégias } \\
\text { para lidar com vários } \\
\text { sintomas físicos e } \\
\text { psicológicos entre } \\
\text { pacientes com câncer } \\
\text { de pulmão sintomático } \\
\text { avançado e seus } \\
\text { principais cuidadores. }\end{array}$ & $\begin{array}{l}\text { Entrevistas } \\
\text { qualitativas } \\
\text { semiestruturadas } \\
\text { individuais, } \\
\text { enquadrando } \\
\text { estresse e } \\
\text { enfrentamento. }\end{array}$ & $\begin{array}{l}21 \text { pacientes com } \\
\text { câncer de pulmão } \\
\text { sintomático } \\
\text { avançado e } \\
\text { cuidadores } \\
\text { familiares } \\
\text { primários. }\end{array}$ & $\begin{array}{l}\text { Os entrevistados explanaram } \\
\text { que continuaram mantendo suas } \\
\text { rotinas e procuraram familiares } \\
\text { e amigos para suporte para lidar } \\
\text { com os sintomas, tendo alterações } \\
\text { na eficácia com frequência. O } \\
\text { apoio de profissionais da área da } \\
\text { saúde também complementou } \\
\text { favoravelmente, além de outras } \\
\text { formas de enfrentamento como } \\
\text { religião, positividade, desvio } \\
\text { de pensamentos relacionados } \\
\text { à enfermidade e mudança de } \\
\text { expectativas. }\end{array}$ & IV \\
\hline $\begin{array}{l}\text { SORATO, D. B. } \\
\text { OSÓRIO, F. L. } \\
\text { (2015), Reino Unido. }\end{array}$ & $\begin{array}{l}\text { Analisar a } \\
\text { desesperança, } \\
\text { ansiedade, depressão } \\
\text { e QV de pacientes } \\
\text { com câncer avançado, } \\
\text { antes da primeira } \\
\text { visita no ambulatório } \\
\text { de cuidados paliativos } \\
\text { e depois no início do } \\
\text { acompanhamento } \\
\text { médico, examinando } \\
\text { a existência de } \\
\text { estratégias de } \\
\text { enfrentamento. }\end{array}$ & $\begin{array}{l}\text { Foram utilizados } \\
\text { instrumentos } \\
\text { autoaplicáveis: } \\
\text { a BHS, EORTC- } \\
\text { QLQ-C30, a HADS } \\
\text { e Inventário de } \\
\text { Estratégias de } \\
\text { Enfrentamento de } \\
\text { Folkman e Lazarus. }\end{array}$ & $\begin{array}{l}85 \text { pacientes, } \\
\text { sendo } 56,5 \% \text { do } \\
\text { sexo feminino com } \\
\text { câncer avançado } \\
\text { sem opções } \\
\text { terapêuticas } \\
\text { curativas. }\end{array}$ & $\begin{array}{l}\text { Os escores de desesperança, } \\
\text { ansiedade, depressão e QV foram } \\
\text { invariáveis, mas os escores de } \\
\text { fadiga e de dor diminuíram, e o } \\
\text { comprometimento social aumentou; } \\
\text { o estudo mostra também que } \\
\text { o apoio social e a reavaliação } \\
\text { positiva foram usados como } \\
\text { estratégia de enfrentamento, sendo } \\
\text { contrariamente correspondidos com } \\
\text { a desesperança. }\end{array}$ & III \\
\hline $\begin{array}{l}\text { NIPP, R. D. et } \\
\text { al.(2016), Estados } \\
\text { Unidos. }\end{array}$ & $\begin{array}{l}\text { Relacionar estratégia } \\
\text { de enfrentamento } \\
\text { com QV e humor em } \\
\text { pacientes com câncer } \\
\text { incurável. }\end{array}$ & $\begin{array}{l}\text { Estudo randomizado } \\
\text { de cuidados } \\
\text { paliativos usando } \\
\text { FACT-G, HADS, e } \\
\text { Brief COPE. }\end{array}$ & $\begin{array}{l}350 \text { pacientes } \\
\text { dentro de } 8 \\
\text { semanas após } \\
\text { o diagnóstico de } \\
\text { incurável câncer } \\
\text { de pulmão ou } \\
\text { gastrointestinal } \\
\text { e antes da } \\
\text { randomização. }\end{array}$ & $\begin{array}{l}\text { A utilização de suporte emocional } \\
\text { e de aceitação como táticas de } \\
\text { enfrentamento teve ligação com } \\
\text { melhor QV e humor. Já a negação } \\
\text { e auto culpa teve uma correlação } \\
\text { negativa com os resultados. }\end{array}$ & I \\
\hline $\begin{array}{l}\text { HAGAN, T. L. et } \\
\text { al. (2017), Estados } \\
\text { Unidos. }\end{array}$ & $\begin{array}{l}\text { Validar sete sub } \\
\text { escalas do Brief } \\
\text { COPE em uma grande } \\
\text { amostra de pacientes } \\
\text { recém-diagnosticados } \\
\text { com câncer incurável } \\
\text { de pulmão e câncer } \\
\text { gastrointestinal não } \\
\text { colo retal. }\end{array}$ & $\begin{array}{c}\text { Ensaio clínico } \\
\text { randomizado de } \\
\text { cuidados paliativos } \\
\text { precoces. }\end{array}$ & 350 pacientes. & $\begin{array}{l}\text { Foram identificadas estratégias } \\
\text { positivas, como: aceitação, } \\
\text { reformulação positiva apoio } \\
\text { emocional. Além disso, aspectos } \\
\text { negativos, tais como: culpa, } \\
\text { negação e desengajamento } \\
\text { comportamental. }\end{array}$ & I \\
\hline
\end{tabular}




\begin{tabular}{|c|c|c|c|c|c|}
\hline $\begin{array}{l}\text { KESTENBAUM, } \\
\text { A. et al. (2017), } \\
\text { Estados Unidos. }\end{array}$ & $\begin{array}{l}\text { Avaliar a viabilidade } \\
\text { e aceitabilidade dos } \\
\text { cuidados espirituais } \\
\text { feitos pelo capelão em } \\
\text { pacientes com câncer } \\
\text { avançado em paliação } \\
\text { ambulatorial. }\end{array}$ & $\begin{array}{l}\text { Estudo qualitativo } \\
\text { com utilização } \\
\text { da Brief RCOPE, } \\
\text { FACIT-Sp-12, Mini- } \\
\text { MAC. }\end{array}$ & $\begin{array}{l}21 \text { pacientes em } \\
\text { cuidados paliativos } \\
\text { e } 3 \text { capelães. }\end{array}$ & $\begin{array}{l}\text { Foram encontrados aumentos } \\
\text { significativos na sub escala "fé" do } \\
\text { FACIT-Sp-12, a sub escala "espírito } \\
\text { de luta" do mini-MAC e fator de } \\
\text { coping adaptativo Mini-MAC. Duas } \\
\text { tendências foram observadas: um } \\
\text { aumento no positivo enfrentamento } \\
\text { religioso no Brief RCOPE e } \\
\text { aumento do fatalismo (uma sub } \\
\text { escala do Mini-MAC). }\end{array}$ & IV \\
\hline $\begin{array}{l}\text { MATOS, T. D. S. et } \\
\text { al.(2017), Brasil. }\end{array}$ & $\begin{array}{l}\text { Avaliar a QV e coping } \\
\text { religioso-espiritual } \\
\text { em pacientes sob os } \\
\text { cuidados paliativos } \\
\text { oncológicos. }\end{array}$ & $\begin{array}{l}\text { Estudo transversal, } \\
\text { com utilização do } \\
\text { MQOL e o CRE } \\
\text { breve. }\end{array}$ & $\begin{array}{l}96 \text { pacientes de } \\
\text { ambulatório de } \\
\text { cuidados paliativos } \\
\text { e } 96 \text { voluntários } \\
\text { saudáveis. }\end{array}$ & $\begin{array}{l}\text { A maioria dos participantes } \\
\text { demonstraram boa QV e alta utilização } \\
\text { do CRE, porém os pacientes em } \\
\text { cuidados paliativos utilizaram mais } \\
\text { o fator negativo. Houve melhor } \\
\text { percepção da QV nos participantes } \\
\text { do sexo masculino, católicos e com } \\
\text { maiores escores no CRE. }\end{array}$ & III \\
\hline $\begin{array}{l}\text { ROHDE, G. et al. } \\
\text { (2017), Noruega. }\end{array}$ & $\begin{array}{l}\text { Explorar o bem-estar } \\
\text { espiritual em pacientes } \\
\text { com câncer colo } \\
\text { retal que receberam } \\
\text { quimioterapia na fase } \\
\text { paliativa. }\end{array}$ & $\begin{array}{l}\text { Método qualitativo } \\
\text { de entrevistas em } \\
\text { profundidade e } \\
\text { uma abordagem de } \\
\text { edição hermenêutica } \\
\text { para as análises e } \\
\text { interpretações. }\end{array}$ & $\begin{array}{c}20 \text { pacientes com } \\
\text { idades entre } 34 \text { e } \\
75 \text { anos. }\end{array}$ & $\begin{array}{l}\text { Os pacientes demonstraram } \\
\text { mudanças em seus objetivos para } \\
\text { outras metas alcançáveis na tentativa } \\
\text { de aumentar seu bem-estar espiritual. } \\
\text { Em termos de seus relacionamentos } \\
\text { com os outros e com Deus ou um } \\
\text { poder maior, pareciam enfatizar } \\
\text { relacionamentos importantes antes } \\
\text { de ficarem mais doentes. }\end{array}$ & IV \\
\hline $\begin{array}{l}\text { RIBEIRO, M. S. et } \\
\text { al. (2018), Brasil. }\end{array}$ & $\begin{array}{l}\text { Identificar as } \\
\text { percepções de idosos } \\
\text { em cuidados paliativos } \\
\text { sobre o enfrentamento } \\
\text { da doença e do } \\
\text { envelhecimento. }\end{array}$ & $\begin{array}{l}\text { Estudo exploratório, } \\
\text { transversal, } \\
\text { descritivo, } \\
\text { qualitativo. }\end{array}$ & $\begin{array}{l}\text { A análise compõe } \\
11 \text { idosos } \\
\text { em cuidados } \\
\text { paliativos. }\end{array}$ & $\begin{array}{l}\text { Foram identificados dois eixos: o } \\
\text { primeiro, resistir para sobreviver } \\
\text { e viver, e o segundo, resistir para } \\
\text { morrer bem. O primeiro possui } \\
\text { duas categorias: envelhecimento } \\
\text { com integridade e desenvolvimento } \\
\text { resiliente. O segundo eixo, possui } \\
\text { apenas uma categoria e refere-se } \\
\text { às percepções dos idosos sobre o } \\
\text { estresse relacionado à doença. }\end{array}$ & III \\
\hline $\begin{array}{l}\text { SERPENTINI, S. et } \\
\text { al. (2019), Itália. }\end{array}$ & $\begin{array}{l}\text { Avaliar a auto eficácia } \\
\text { no enfrentamento do } \\
\text { câncer em cuidados } \\
\text { paliativos. }\end{array}$ & $\begin{array}{l}\text { CBI-B/I foi } \\
\text { administrado em } \\
\text { conjunto com } \\
\text { EORTC QLQ-C30, } \\
\text { Mini-MAC, CCL e } \\
\text { HADS. }\end{array}$ & $\begin{array}{l}216 \text { pacientes } \\
\text { com câncer } \\
\text { avançado que } \\
\text { se apresentaram } \\
\text { em clínicas de } \\
\text { cuidados paliativos. }\end{array}$ & $\begin{array}{l}\text { A análise evidenciou melhor } \\
\text { gerenciamento de estresse de } \\
\text { enfrentamento, por meio de } \\
\text { estratégias como: espírito de } \\
\text { luta, esquecimento cognitivo e o } \\
\text { fatalismo. }\end{array}$ & II \\
\hline $\begin{array}{l}\text { WARMENHOVEN, } \\
\text { F. et al. (2019), } \\
\text { Holanda. }\end{array}$ & $\begin{array}{l}\text { Explorar as opiniões } \\
\text { dos pacientes em } \\
\text { cuidados paliativos } \\
\text { sobre os recursos } \\
\text { e formas de } \\
\text { enfrentamento que os } \\
\text { ajudam a prevenir ou } \\
\text { controlar os sintomas } \\
\text { de humor. }\end{array}$ & $\begin{array}{l}\text { Estudo piloto } \\
\text { qualitativo } \\
\text { realizado por meio } \\
\text { de entrevistas } \\
\text { semiestruturadas } \\
\text { presenciais. }\end{array}$ & $\begin{array}{l}\text { Amostra composta } \\
\text { por } 15 \text { pacientes } \\
\text { em cuidados } \\
\text { paliativos } \\
\text { ambulatoriais. }\end{array}$ & $\begin{array}{l}\text { Foram identificados recursos de } \\
\text { enfrentamento relacionados ao } \\
\text { paciente, como positividade e } \\
\text { espiritualidade; à rede social de } \\
\text { apoio, tais como buscar apoio familiar, } \\
\text { conjugal, comunitário e religioso; e } \\
\text { relacionados ao apoio profissional } \\
\text { recebido, como perguntas frequentes } \\
\text { sobre seu bem estar. }\end{array}$ & IV \\
\hline
\end{tabular}

Legenda: Avaliação Funcional de Terapia de Doença Crônica e Espiritual (FACIT-Sp-12), Coping Orientation to Problem Experienced (Brief CO$P E)$,Beck HopelessnessScale (BHS), BriefReligiousCopingScale (Brief RCOPE), CancerBehaviorlnventory-Brief (CBI-B/I), CancerConcernsChecklist (CCL), Coping Religioso/Espiritual (CRE), Escala de Ajuste Mental ao Câncer (MAC), Escala de Avaliação Funcional da Terapia do Câncer - Geral (FACT-G), Escala Visual Analógica (EVA), EuropeanOrganization for ResearchandTreatmentofCancer (EORTC-QLQ-C30), Hospital AnxietyandDepressionScale (HADS),McGillQualityof Life Questionnaire (MQOL), Mini-Ajuste Mental ao Câncer (Mini-MAC), Qualidade de Vida (QV). Fonte: próprios autores, 2019. 


\section{DISCUSSÃO}

Os estudos utilizados apresentavam diferenças no que diz respeito aos objetivos, metodologia e instrumentos utilizados para avaliar as estratégias de enfrentamento do câncer em cuidados paliativos, o que não permitiu qualquer forma de generalização.

Algumas produções bibliográficas apontam o coping religioso/espiritual como a principal estratégia de enfrentamento do câncer, com destaque para o coping religioso/ espiritual negativo, sendo fator adjuvante na percepção da qualidade de vida dos pacientes em cuidados paliativos, evidenciando que o enfrentamento não está condicionado apenas a fatores positivos ${ }^{(16)}$.

Seguindo essa mesma idéia, autores relatam em seus estudos que a espiritualidade e cuidados paliativos são dois pilares essenciais a qualidade de vida e que, a espiritualidade é o refúgio dos pacientes que necessitam de cuidados paliativos não só para o alívio das dores físicas, mas das dores emocionais e relacionamentos envolvidos no processo do cuidado ${ }^{(26-27)}$.

Partindo dessa premissa, os pacientes oncológicos em cuidados paliativos, apresentam mudanças em seus objetivos para outras metas alcançáveis na tentativa de aumentar seu bem-estar espiritual. Essa hipótese é defendida ${ }^{(18)}$ alegando ainda que, em termos de seus relacionamentos com os outros e com Deus ou um poder maior, os pacientes fora de possibilidade terapêutica, parecem enfatizar o estabelecimento de relacionamentos importantes antes de ficarem mais doentes, trazendo à tona um tipo de "paliação precoce".

Em contrapartida, outros estudos ${ }^{(22)}$, se referem que a principal forma de enfrentamento está relacionada aos recursos e as formas de enfrentamento diretamente relacionadas ao paciente, tais como: resolução ativa de problemas, convivendo com suas limitações de maneira flexível, distraindo-se, cuidando de si mesmos, realizando diversas (físicas) atividades e viver a vida o mais normal possível. Seguidas dos recursos relacionados à rede social do paciente, como família, ambiente social e amigos e, por último, a relevância da espiritualidade.

Para outros estudiosos na área ${ }^{(28)}$ as fontes de força e motivação para o enfrentamento do câncer é o apoio social destacado como sinal de carinho e cuidado, que minimiza o sentimento de solidão, seguida de ser convivente com outras pessoas que estão na mesma situação de doença onde emerge sentimentos de esperança e força para lutar e a religiosidade onde a fé também é referida como fonte de força e motivação.

No tocante as estratégias positivas, sendo elas: apoio emocional, aceitação e reformulação positiva e ativa, e as negativas, negação, auto culpa e desengajamento comportamental. Os autores propõem ainda a existência de associação positiva com mais tipos de enfrentamento para evitar, como por exemplo, a culpa e o desengajamento comportamental (16).

A importância das emoções no enfrentamento do câncer é ratificada para uma melhor qualidade de vida. Acrescentam também outras estratégias, como a alta utilização de apoio emocional e aceitação. Porém, no mesmo estudo, teve um alto índice de negação e auto culpa como enfretamento, resultando em uma pior qualidade de vida e humor ${ }^{(17)}$.

Os esforços cognitivos e comportamentais são estratégias de enfrentamento, ou coping para administrar as demandas específicas internas e externas causadas por situações estressantes, que $\mathrm{o}$ enfrentamento pode ter seu foco voltado ao problema, quando a pessoa procura a resolução da situação por meio de informações sobre um caso estressante e, assim, analisa a ação que acredita ser mais eficaz para solucionar esse fato estressante; ou ainda pode ser focado na emoção, quando as estratégias adotadas são carregadas de emoção, que ocasionam processos de autodefesa que servem como escudo, dificultando o confronto com o fator estressante. Deste modo, o indivíduo pode se distanciar, fugir ou se esquivar do problema.Além disso, seu estudo relata também sobre essas estratégias de enfrentamento relacionado com a ansiedade. Onde pacientes que possuem baixo nível de ansiedade tendem a utilizar como estratégia a resolução dos problemas e quando o nível é médio a alto o enfrentamento com foco na emoção ${ }^{(29)}$.

Cabe ressaltar que as formas de enfrentamento do câncer em cuidados paliativos estão associadas à qualidade de vida do paciente, fatores estes relacionados ao enfrentamento ou coping espiritual, enquanto SORATO e OSÓRIO (2015) abordam a importância do apoio social e da positividade ${ }^{(15 ; 21 ; 24)}$.

Os aspectos como apoio familiar e amigos tem sido utilizados pelos pacientes como formas de lidar com os sintomas físicos e psicológicos decorrentes do câncer fora de possibilidade terapêutica. Os autores ainda acrescentam que o apoio de profissionais da área da saúde contribuiu significativamente para melhor enfrentamento da doença oncológica ${ }^{(23 ; 30)}$.

A abordagem do entendimento do envelhecimento, junto com uma enfermidade, abrange resiliência, uma vez que os componentes do estudo focaram em suas conquistas ao invés de suas privações ${ }^{(20)}$. Dessa maneira, fizeram o uso de apoio espiritual, reestruturação cognitiva e aceitação como meios de enfrentamento.

Os pacientes em cuidados paliativos adotam como enfrentamento um instinto de combatente (espírito de luta) e o fatalismo para enfrentarem a enfermidade. Entretanto, somente outras evidências trazem consigo a estratégia de esquecimento cognitivo. Os autores correlacionam essa busca com a melhoria no gerenciamento do estresse de enfrentamento ${ }^{(19 ; 25)}$ 


\section{CONCLUSÃO}

Os pacientes em cuidados paliativos apresentaram como principais estratégias de enfrentamento do câncer, o coping religioso/espiritual, o apoio familiar, e a rede de apoio social (comunidade, família, etc.). Destes, destacase que o fator espiritual tem sido utilizado pela maioria dos pacientes em cuidados paliativos, e que, o mesmo é correlacionado com a qualidade de vida.

Essas relações entre o enfrentamento espiritual/ religioso e cuidados paliativos têm sido cada vez mais investigadas e as evidências apontam para uma relação, na maioria das vezes, positiva. No entanto, a relação entre qualidade de vida e o coping religioso/espiritual, em cuidados paliativos, ainda é tema pouco abordado na literatura, apesar de sua importância.

Observando os estudos, entende-se que existe um arcabouço considerável e explorável de ações por parte do enfermeiro no que diz respeito ao manejo do quadro paliativo, onde a religião e espiritualidade devem ser fortalecidas pela equipe multiprofissional.

É notório que o profissional de enfermagem está em constante contato com os pacientes cujo câncer está fora de possibilidade terapêutica, permitindo o estabelecimento de vínculos e uma análise crítica e clínica do quadro paliativo, assim como a identificação das estratégias de enfrentamento.

Ressalta-se ainda que os resultados obtidos nesta revisão integrativa possam contribuir acerca do conhecimento a respeito do enfrentamento do câncer em cuidados paliativos e suas implicações nas intervenções de enfermagem.

Sugere-se a execução de estudos de campo com o objetivo de identificar, de forma prática, as formas de enfrentamento do câncer fora de possibilidade terapêutica e evidenciar a importância de aplicá-las no Processo de Enfermagem. 


\section{REFERÊNCIAS}

I. Oliveira JM, Reis JB, Silva RA. Busca por Cuidado Oncológico: Percepção de Pacientes e Familiares. Revista de Enfermagem Ufpe OnLine, [Internet]. 2018 [acesso 10 de setembro de 2019]; I2(4):938-947. Disponível em: <http://dx.doi. org/| 0.5205/| 98|-8963-v| 2i4a23 |359p938-946-20|8>. Acesso em: 10 de setembro de 2019.

2. Silva RS et al. Nursing Team Actions From The Perspective Of Families Of Patients Under Palliative Care. Reme: Revista Mineira de Enfermagem [Internet]. 2016 [acesso em 20 de setembro de 2019]; 20:I-9. Disponível em: <http:// dx.doi.org//0.5935//4I5-2762.20160053>. Acesso em: 20 de setembro de 2019.

3. Freire MEM, Costa SFG, Lima RAG, Sawada NO. Qualidade de vida relacionada à saúde de pacientes com câncer em cuidados paliativos. Texto contexto - enferm. [Internet]. 2018 [acesso em 14 de março de 2020]; 27(2). Disponível em: https://doi.org/l0.1590/0104-070720180005420016.

4. Picollo DP, Fachini M.A atenção do enfermeiro ao paciente em cuidado paliativo. Rev Ciênc Med. [Internet]. 2018 [acesso em I4 março de 2020];27(2):85-92. Disponível em: http://dx.doi. org/I0.24220/23 I8-0897v27n2a3855.

5. Izoo JM, Cunha AMR, Cesarino CB, Martins MRIM. The impact of chronic pain on the quality of life and on the functional capacity of cancer patients and their caregivers. BrJP [Internet]. 2019 [acesso em I 3 de março de 2020]; 2(4):336-4I. Disponível em: https://doi.org//0.5935/25950118.20190062 .

6. Matos $\mathrm{K}$ et al. Estratégias de Enfrentamento do Câncer Adotadas por Familiares de Indivíduos em Tratamento Oncológico. Revista Psicologia e Saúde [Internet]. 2016 [acesso em 25 de outubro de 2019]; I(8):I-16. Disponível em: $<$ http://dx.doi.org/10.20435/2177093×2016I0I>. Acesso em: 25 de outubro de 2019.

7. Costa DT, Silva DMR, Cavalcanti IDL, Gomes ET, Vasconcelos JLA, Carvalho MVG. Religious/spiritual coping and level of hope in patients with cancer in chemotherapy. Rev Bras Enferm [Internet]. 2019 [acesso em 12 de março de 2020];72(3):640-5. Disponível em: doi: http://dx.doi. org/10.1590/0034-7I67-2018-0358.

8. Hyde MK et al. Psycho-oncology assessment in Chinese populations: a systematic review of quality of life and psychosocial measures. European Journal of Cancer Care, [s.l.] [Internet]. 2015 [acesso em 25 de outubro de 2019]; 25(5):69|-7/8, Disponível em:<http://dx.doi.org/I0.1 III/ ecc. 12367>.

9. Oliveira TR, Martins BCT, Rocha ME, Gomes NS, Sena Aires VGF. Sistematização da Assistência de Enfermagem: análise da produção científica em oncologia - revisão integrativa. Braz. J. of Develop. [Internet]. 2020 [acesso em 14 de março de 2020]; 6(2): 954I-9555 Disponível em:. https://doi. org/| 0.34II7/bjdv6n2-3|4.

10. Rolim DS, Arboit EL, Kaffer CT, Marisco N, Ely GZ, Arboit
J. Produção científica de enfermeiros brasileiros sobre enfermagem e oncologia: revisão narrativa da literatura. Arq. Cienc. Saúde UNIPAR [Internet]. 2019 [acesso em 12 de março de 2020]; 23(I): 4 I-47, jan./set. 2019. Disponível em: https://doi.org/10.25I I0/arqsaude.v23il.2019.626I.

I I. Peiter CC, Caminha MEP, Lanzoni GMM, Erdmann AL. Gestão do cuidado de enfermagem ao paciente oncológico num hospital geral: uma Teoria Fundamentada nos Dados. Revista de Enfermagem Referência [Internet]. 2016 [acesso em 10 de março de 2020]; I I. Disponível em: http://dx.doi. org/I0.12707/RIVI6044.

12. Almeida PF, Barbosa MGA, Santos SM, Silva El, Lins SRO. A relação entre o enfermeiro e o paciente nos cuidados paliativos oncológicos. Braz.J. Hea. Rev. [Internet]. 2020 [acesso em 14 de março de 2020] 3(2):|465-|483. Disponível em: https://doi.org/l0.34 I 19/bjhrv3n2-0II.

13. Santos CMC, Pimenta CAM, Nobre MRC. The PICO strategy for the research question construction and evidence search. Revista Latino-americana de Enfermagem, [s.l.] [Internet]. 2007 [acesso em 10 de setembro de 2019]; I5(3). Disponível em: <http://dx.doi.org/I0.1590/s0104I |692007000300023>.

14. BotelhoLLR, CunhaCCA, MacedoM. Método da revisão integrativa nos estudos organizacionais. Gestão e Socieda-de [Internet].20 II [acesso em 26 mai 20I6]; 5 (I I): I 2I-I36. Disponível em: https://www.gestaoesociedade.org/gestaoesociedade/article/view//220/906

15. Galvão TF, Pereira MG. Avaliação da qualidade da evidência de revisões sistemáticas. Epidemiologia e Serviços de Saúde, [s.l.] [Internet]. 2015 [acesso em 04 de novembro de 2019]; 24(I): 75-778, Disponível em: <http://dx.doi. org/l0.5123/s I679-49742015000100019.

16. Mattos TDS et al. Quality of life and religious-spiritual coping in palliative cancer care patients. Revista Latino-americana de Enfermagem, [s.l.] [Internet]. 2017 [acesso em 21 de outubro de 2019]; 25:I-9. Disponível em: <http://dx.doi. org/10.1590/1518-8345.1857.2910>.

17. Hagan TL et al. Coping in Patients With Incurable Lung and Gastrointestinal Cancers: A Validation Study of the Brief COPE. Journal of Pain and Symptom Management, [s.l.] [Internet]. 2017 [acesso em 19 de outubro de 2019]; 53(I):|3|-|38. Disponível em:<http://dx.doi.org//0.1016/j. jpainsymman.2016.06.005>.

18. Nipp RD et al.The relationship between coping strategies,quality of life, and mood in patients with incurable cancer. Cancer, [s.l.] [Internet]. 2016 [acesso em 10 de setembro de 2019]; I22(|3):2II0-21/6. Disponível em: <http://dx. doi.org/10.1002/cncr.30025.

19. Rohde $G$ et al. Bem-estar espiritual em pacientes com câncer colorretal metastático recebendo quimioterapia não curativa: um estudo qualitativo. Enfermagem em Câncer [Internet]. 2017 [acesso em 10 de setembro de 2019]; 40(3):209-2/6. Disponível em: <https://insights.ovid.com/ 
crossref?an=00002820-20 I 705000-00007>.

20. Kestenbaum A et al. What Impact Do Chaplains Have? A Pilot Study of Spiritual AIM for Advanced Cancer Patients in Outpatient Palliative Care. Journal of Pain and Symptom Management, [s.l.] [Internet]. 2017 [acesso em 25 de outubro de 2019]; 54(5): 707-7|4. Disponível em:<http://dx.doi. org/10.1016/j.jpainsymman.2017.07.027.

2I. Ribeiro MS. Borges MS. Perceptions of aging and falling ill: a study with elderly persons in palliative care. Revista Brasileira de Geriatria e Gerontologia, [s.I.] [Internet]. 2018 [acesso em 10 de setembro de 20I8]; 2I(6):70I710. Disponível em: <http://dx.doi.org//0.1590//98I22562018021.180139.

22. Bovero A et al. Spirituality, quality of life, psychological adjustment in terminal cancer patients in hospice. European Journal of Cancer Care, [s.l.] [Internet]. 2015 [acesso em 19 de outubro de 2019]; 25(6): 961-969. Disponível em:<http://dx.doi.org/10.1 I I I/ecc. 12360>.

23. Warmenhoven F et al. 'Life is still worth living': a pilot exploration of self-reported resources of palliative care patients. Bmc Family Practice, [s.l.] [Internet]. 2016 [acesso em 20 de outubro de 2019]; 17(I): I-6. Disponível em: <http://dx.doi.org/ | 0.1 |86/s | 2875-0 |6-0450-y>

24. Mosher CE et al. Coping with physical and psychological symptoms: a qualitative study of advanced lung cancer patients and their family caregivers. Supportive Care in Cancer, [s.l.] [Internet]. 2014 [acesso em 10 de setembro de 2019]; 23(7):2053-2060. Disponível em: <http://dx.doi. org//0.1007/s00520-0|4-2566-8>.

25. Serpentini $S$ et al. Self-efficacy for coping: utility of the Cancer behavior inventory (Italian) for use in palliative care.
Bmc Palliative Care, [s.l.] [Internet]. 2019 [acesso em 13 de setembro de 2019]; I8(I):I-9. Disponível em: <http:// dx.doi.org/| 0. I |86/s | 2904-0 |9-0420-y>.

26. Olivera IC et al. Cuidados paliativos e espiritualidade no Sistema Único de Saúde: Uma Revisão sistemática da literatura. Revista Multidisciplinar e de Psicologia [Internet]. 2019 [acessp em 4 de novembro de 2019]; I3(45): 405-4I9, 2019. Disponível em: <http://idonline.emnuvens.com.br/id> 27. Evangelista CB, Lopes MEL et al. Cuidados paliativos e espiritualidade: revisão integrativa da literatura. Revista Brasileira de enfermagem [Internet]. 2016 [acesso em 02 de novembro de 2019]; 69(3):59I-60I. Disponível em: www.scielo.br/ pdf/reben/v69n3/0034-7167-reben-69-03-059I.pdf.

28. Teston EF et al Feelings and difficulties experienced by cancer patients along the diagnostic and therapeutic itineraries. Esccola Ana Nery [Internet]. 2018 [acesso em 12 de outubro de 2019]; 22(4): I-8. Disponível: http://www.scielo. br/pdf/ean/v22n4/|4|4-8|45-ean-22-04-e20 I800 I7.pdf.

29. Silva AV, Zandonade E, Amorim MHC. Ansiedade e o enfrentamento de mulheres com câncer de mama em quimioterapia. Revista Latino Americano de Enfermagem [Internet]. 2017 [acesso em 5 de novembro de 2019]; 25: I-7. Disponível em:<http://www.scielo.br/pdf/rlae/v25/ pt_0104-I 169-rlae-25-e289l.pdf>.

30. Ferreira MLSM et al. Ser cuidador de familiar com câncer. Ciencia y Enfermeira [Internet]. 2018 [acesso em 14 de março de 2020]; 24: 6. Disponível em: http://dx.doi. org/10.4067/s07/7-95532018000100206.

Recebido: 2020-03-14

Aceito: $2020-07-15$ 